\title{
(- OPEN ACCESS \\ How well can poor child health and development be predicted by data collected in early childhood?
}

\author{
Viviane S Straatmann, ${ }^{1}$ Anna Pearce, ${ }^{2}$ Steven Hope, ${ }^{3}$ Benjamin Barr, \\ Margaret Whitehead, ${ }^{1}$ Catherine Law, ${ }^{3}$ David Taylor-Robinson ${ }^{1}$
}

\begin{abstract}
- Additional material is published online only. To view please visit the journal online (http://dx.doi.org/10.1136/ jech-2018-211028)

'Department of Public Health and Policy, University of Liverpool, Liverpool, UK ${ }^{2} \mathrm{MRC} / \mathrm{CSO}$ Social and Public Health Sciences Unit, Institute of Health and Wellbeing, University of Glasgow, Glasgow, UK ${ }^{3}$ Population, Policy and Practice, UCL Great Ormond Street Institute of Child Health, London, UK
\end{abstract}

Correspondence to Dr Viviane S Straatmann, Department of Public Health and Policy, University of Liverpool, Liverpool L69 3GB, UK; v.schultz-straatmann@liv. ac.uk

Received 15 May 2018 Revised 14 August 2018 Accepted 30 August 2018 Published Online First 21 September 2018

\section{A Check for updates}

(c) Author(s) (or their employer(s)) 2018. Re-use permitted under CC BY. Published by BMJ.

To cite: Straatmann VS, Pearce A, Hope S, et al. $J$ Epidemiol Community Health 2018:72:1132-1140.

\section{ABSTRACT \\ Background Identifying children at risk of poor} developmental outcomes remains a challenge, but is important for better targeting children who may benefit from additional support. We explored whether data routinely collected in early life predict which children will have language disability, overweight/obesity or behavioural problems in later childhood.

Methods We used data on 10262 children from the UK Millennium Cohort Study (MCS) collected at 9 months, 3 , and 11 years old. Outcomes assessed at age 11 years were language disability, overweight/obesity and socioemotional behavioural problems. We compared the discriminatory capacity of three models: (1) using data currently routinely collected around the time of birth; (2) Model 1 with additional data routinely collected at 3 years; (3) a statistically selected model developed using a larger set of early year's risk factors for later child health outcomes, available in the MCS - but not all routinely collected. Results At age 11, $6.7 \%$ of children had language disability, $26.9 \%$ overweight/obesity and $8.2 \%$ socioemotional behavioural problems. Model discrimination for language disability was moderate in all three models (area under the curve receiver-operator characteristic $0.71,0.74$ and 0.76 , respectively). For overweight/obesity, it was poor in model $1(0.66)$ and moderate for model $2(0.73)$ and model $3(0.73)$. Socioemotional behavioural problems were also identified with moderate discrimination in all models $(0.71 ; 0.77 ; 0.79$, respectively).

Conclusion Language disability, socioemotional behavioural problems and overweight/obesity in UK children aged 11 years are common and can be predicted with moderate discrimination using data routinely collected in the first 3 years of life.

\section{BACKGROUND}

The antenatal period and first $2-3$ years are crucial stages that influence children's subsequent development and health outcomes. By age 3 years, many physical, cognitive and emotional development problems are apparent, but there remain opportunities to intervene to improve child outcomes. ${ }^{1-3}$ There is increasing recognition of the need to collect better early years' data to identify children most at risk early, in order to facilitate more appropriate referral to services and early intervention programmes. ${ }^{4}$ Accordingly, the National Health Service (NHS) in England has been developing an improved national maternity services dataset, to collate routinely collected sociodemographic and perinatal information. In addition, in 2015, a new 'integrated universal health check' was introduced for children aged 2-3 years in England to provide a more complete picture of children's health and development. $^{3-5}$

A central challenge in using these new datasets is to accurately identify children most in need of additional support to achieve their greater longterm health and developmental potential and then deciding the most appropriate combination of universal and targeted service. ${ }^{6} 7$ Predictive risk models, used widely for applications such as cardiovascular risk prediction, ${ }^{8-10}$ have not been extensively assessed to inform child public health interventions. One previous study using a UK cohort showed that maternal age was a poor predictor of child health and development up to age 5 and that prediction was improved by including data on mother's smoking status during pregnancy, education level, mental health and financial status. ${ }^{11}$ An Australian study using linked early childhood data to identify children with poor development at school entry showed that a model with six perinatal predictors (maternal age, smoking, parity, marital status and both parents' occupation) demonstrated similar discrimination to a model including 22 predictors, constituting a more statistically parsimonious set of perinatal characteristics for predicting developmental vulnerability. ${ }^{12}$

In the context of the new datasets being collected in England, the aim of this study was to explore how early childhood characteristics predict three important developmental outcomes: language disabilities (cognitive outcome), overweight/obesity (physical outcome) and socioemotional behavioural problems (behavioural outcome) in later childhood (11 years). To address this aim, we used data from the UK Millennium Cohort Study (MCS), a nationally representative study of infants born in the early 2000s in the UK, which provides a rich data source on the social context and measures of health for children growing up in the UK. ${ }^{13}$ We assess the predictive capacity of a model using data routinely collected in maternity services; determine how the model's performance improves when this is updated with information collected at age 2-3 years and compare the performance of the enhanced model with a third model using a larger range of early life risk factors for adverse child health collected in MCS.

\section{METHODS}

\section{Data source and study population}

The MCS is a nationally representative sample of children born in the UK between September 2000 and 
January 2002 and followed up at intervals (sweeps) to the present date. We chose the MCS as it captures a wide range of data on the social context for children growing up in the UK and provides actual measures of both early and late child health outcomes that can be used to develop predictive models.

The MCS study oversampled children living in disadvantaged areas and those with high proportions of ethnic minority groups, and non-response weights were used to address sample attrition. Further information on the cohort and sampling design can be found in the cohort profile. ${ }^{13}$ Interviews were carried out by trained interviewers in the home with the main respondent (usually the mother). We used data from three sweeps when the children were aged 9 months, 3 years and 11 years. Information was collected from 18818 infants $(91 \%$ of the 20646 in the target sample), and analysis was restricted to 18296 singleton children.

\section{Cognitive, physical and behavioural outcomes}

We investigated outcomes at 11 years old, an important transition stage between childhood and adolescence marked by the end of primary school. Cognitive ability was evaluated through the British Ability Scale Second Edition (BAS II) Verbal Similarities test, a validated standardised assessment of verbal reasoning and knowledge, normed for children and adolescents from 3 years to 11 years of age..$^{14}{ }^{15}$ We defined children as having language disability if they scored -1.25 SD below the normed mean score for the sample. ${ }^{16-18}$ Overweight/obesity was derived from the body mass index (BMI), using the age and sex-specific International Obesity Task Force cut-offs. ${ }^{19}$ The Strengths and Difficulties Questionnaire (SDQ-maternal report) was used to assess child socioemotional behaviour. The SDQ is a 25 item measure that asks parents to rate their child's behaviour over the previous 6 months using five subscales: peer problems, conduct disorders, hyperactivity, emotional problems and prosocial behaviour. ${ }^{20}$ As in previous studies, ${ }^{21}{ }^{22}$ we used the total difficulties score (excluding the prosocial behaviour subscale), dichotomised at the validated 'borderline-abnormal' (17-40), cut-off score, indicating socioemotional behavioural problems. ${ }^{20}$

\section{Potential predictors}

We outline predictors used in this study, grouped as perinatal (MCS first sweep-9 months), age 3 years (MCS second sweep) and earlier measures of language, SDQ and BMI at age 3 (figure 1). The full details of the coding of the predictors are provided in the online supplementary material .

\section{Modelling approach}

We developed three models:

Model 1: using variables in the MCS that are also currently collected routinely around the time of birth in maternity services in England (15 items). These data have been collected in the NHS in England and collated in the Maternity Services Data Set from April 2015 onwards. ${ }^{23}$

Model 2: using variables collected in maternity services (model 1) plus additional information collected at age 3 years in MCS which capture the five central domains included in the new integrated 2.5-year-old health check in England: (1) personal, social and emotional development, (2) communication and language, (3) physical health, (4) learning and cognitive development and (v) physical development and self-care) ${ }^{2}$ (23 items (15 items of model 1 plus 8 items)).

Model 3: a model including 30 perinatal, family/environmental and early childhood factors up to age 3 years, selected from risk factors for later child health and development problems identified in studies worldwide. ${ }^{24-29}$ Items included in this model overlap all items of model 1 and 18 items of model 2, since we did not include all variables capturing domains of the Ages and Stages Questionnaire (ASQ) which are represented by other instruments.

We applied a statistical selection to the saturated model (30 items), and a predictive model was developed based on statistical parsimony for each outcome. Figure 1 shows the complete description of items included in each model.

\section{Statistical analyses}

First, we assessed the prevalence (\%) for all potential predictor and outcome variables. Relative risks (RRs) and 95\%CI for outcomes at 11 years were estimated using Poisson regression for all predictors included in models 1, 2 and 3. To develop model 3 , we began with a saturated model containing the full range of 30 variables listed above and then selected a smaller number of variables using forwards and backwards stepwise selection ( $p \leq 0.1$ for inclusion and $p>0.11$ for exclusion). Sampling and

Model 1 (15 items): child's sex and birth weight; mother's ethnicity, age at birth, longstanding disabilities/illness, pre-pregnancy BMI, alcohol consumption and smoking in pregnancy, and symptoms of depression and anxiety at birth; gestational age at birth, breastfeeding initiation, type of delivery, Index of Multiple Deprivation (IMD), language spoke at home, and parent's employment status.

Model 2 (23 items): model $1+$ parent's concerns of child speech, and understanding of child speaking; child's longstanding disabilities/illness, hearing problems, walking ability, language disability, socioemotional behavioural problems, and overweight /obesity at age 3 .

Model 3 (30 items): child's sex and birth weight; maternal education at birth, ethnicity, age at birth, pre-pregnancy BMI, alcohol consumption and smoking in pregnancy, longstanding disabilities/illness, symptoms of depression and anxiety at birth, general health at birth, and mental health disorders at child's age 3; family income, IMD, lone parenthood, number of siblings in household, language spoke at home, gestational age at birth, and breastfeeding initiation; parenting style; parent's employment status, educational disagreements of the child, involvement with child's reading at age 3, relationship-closeness with child at age 3, relationship-conflicts with child at age 3 , help child to practice sports at age 3 , and regular bed times at age 3; child's language disability, social-emotional behavioural problems, and overweight/obese at age 3 .

$>$ Statistical selection:

- Language disability (14 items): child's sex; maternal education at birth, ethnicity, and age at birth; family income, IMD, number of siblings in household, gestational age; parent's educational disagreements of the child, involvement with child reading, and relationship-closeness with child at age 3; child's language disability, socioemotional behavioural problems, and regular bed times at age 3 .

- Overweight/Obesity (14 items): child' sex; maternal education at birth, ethnicity, pre-pregnancy BMI, alcohol consumption and smoking in pregnancy, longstanding disabilities/illness, and general health at birth; IMD, parent's employment status, parenting style, parent's involvement with child reading; child's regular bed times, and overweight/obese at age 3 .

- Socioemotional Behavioural Problems (22 items): child's sex and birth weight; maternal education at birth, ethnicity, age at birth, pre-pregnancy BMI, smoking in pregnancy, longstanding disabilities/illness, symptoms of depression and anxiety at birth, general health at birth, and mental health disorders at age 3 of child; family income, IMD, lone parenthood, language spoke at home, parent's employment status, involvement with child reading, and relationship-conflicts with child at age 3 ; parenting style, child's regular bed times, language disability, and social-emotional behavioural problems at age 3 .

Figure 1 Description of items included in models 1, 2 and 3. 
response weights were not used for receiver-operator characteristic (ROC) analysis.

The predicted probability of poor child development was calculated from these regression models. Predictive risk modelling was performed using a ROC curve which is a graphical plot that illustrates the diagnostic ability of a binary classifier system as its discrimination of true positives (ie, sensitivity) versus the fraction of false positives (ie, 1 -specificity). ${ }^{30}$ For each model, we assessed the probability cut-off point to obtain the optimal maximised probability cut-off using a function of the difference between true positive rate and false positive rate over all possible cut-point values. The optimal maximised cut-off is the point where the sensitivity and specificity curves intersected and classifies most of the individuals correctly. ${ }^{31}$ Area under the receiver operating characteristic curve (AUROC) indicates the model's overall capacity to discriminate between those who have or do not have the outcome. This provides an indication of how well the models perform in terms of the probability that a random pair of one child with the poor outcome and one without would be correctly ranked by the predicted probabilities from the model. A guide for classifying the accuracy of a diagnostic test is AUC values of ' $0.90-1=$ excellent', ' $0.80-0.90=$ good', ' $0.70-0.80=$ moderate', ' $0.60-0.70=$ poor' and ' $0.50-0.60=$ fail'. 3132

The integrated discrimination improvement (IDI) for model 2 compared with model 1 and model 3 with model 2 were also calculated. The IDI assesses discrimination without relying on cut-off points and compares the average difference in predicted risk for children with poor health or development with those which do not have poor health or development. The IDI improvement is greater when the second model correctly assigns individuals to higher or lower probabilities of having the outcome in comparison to the first model. ${ }^{33}$ Calibration's accuracy of the models was assessed using the Hosmer-Lemeshow goodness-of-fit $\chi^{2}$ statistic. In this statistic test, the null hypothesis is that predicted proportion equals the observed proportion within ranked groupings (deciles) of predicted risk and a high $\mathrm{p}$ value suggests good calibration of predicted and observed risk. ${ }^{34}$ Dominance analysis, a method for assessing the relative weight of predictive variables in a multivariable regression, was used to estimate the standardised dominance score (SDS) to rank the importance of each variable in each model. ${ }^{35}$ All analyses were conducted in Stata SE V.13.0 (Stata, 2014).

\section{Multiple imputation}

Multiple imputation by chained equation was performed to impute missing data using the 'mi impute chained' command in Stata SE V.13.0 (Stata, 2014). We used data of predictors and the three outcomes at age 11 to shape the imputation process of the other risk factors included in the three models above (imputed sample, $n=10262$ ). We generated 20 datasets, with 200 iterations per imputed dataset. Results were calculated by averaging the results across the 20 imputed datasets using Rubin's rules. ${ }^{36}$ Results from the imputed sample are reported below and for the complete case sample are provided in the supplementary material.

\section{RESULTS}

At 11 years, 6.7\% (95\% CI 6.3\% to $7.2 \%$ ) of children had language disability; $26.9 \%$ (95\% CI $26.1 \%$ to $27.8 \%$ ) overweight/obesity and $8.2 \%$ (95\% CI $7.6 \%$ to $8.7 \%$ ) had socioemotional behavioural problems. Prevalence of outcomes stratified by risk factors is shown in the online supplementary material.

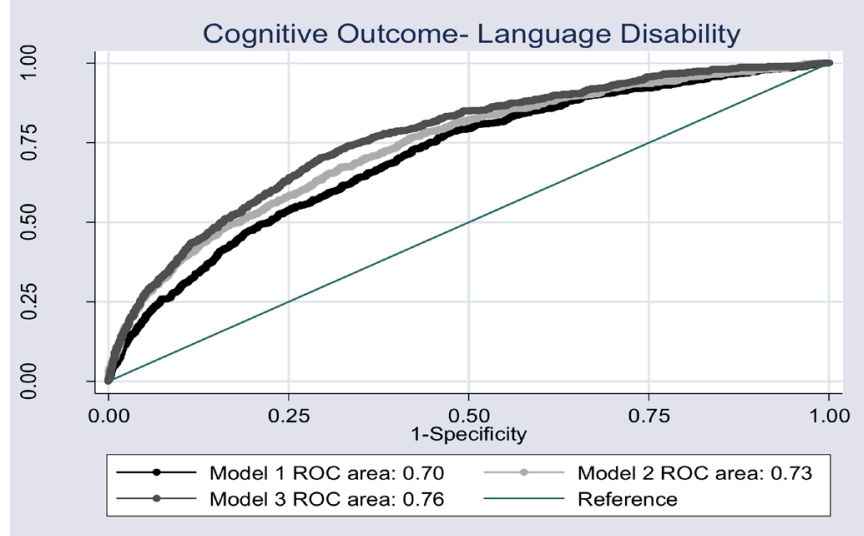

IDI (\%/p value): model 2 over model $13.27 /<0.001$, model 3 over model $21.55 / /<0.001$

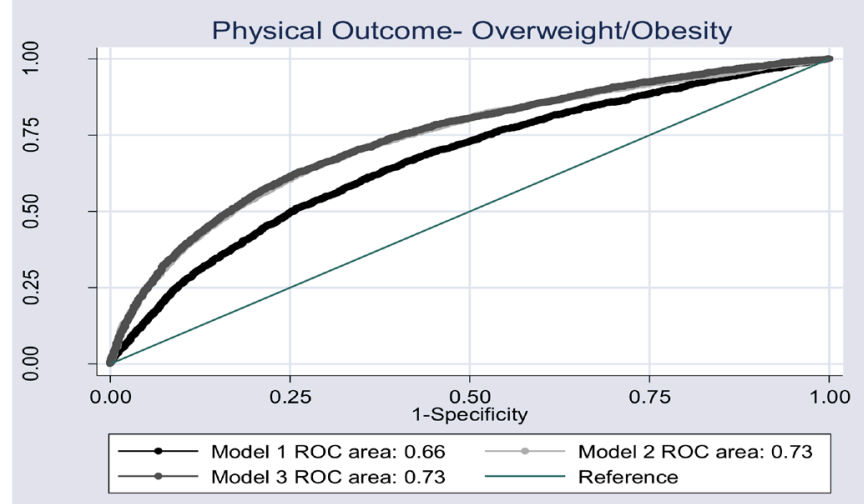

IDI (\%/p value): model 2 over model $18.14 /<0.001$, model 3 over model $20.41 / /<0.001$ Behavioural Outcome- Socioemotional Behavioural Problems

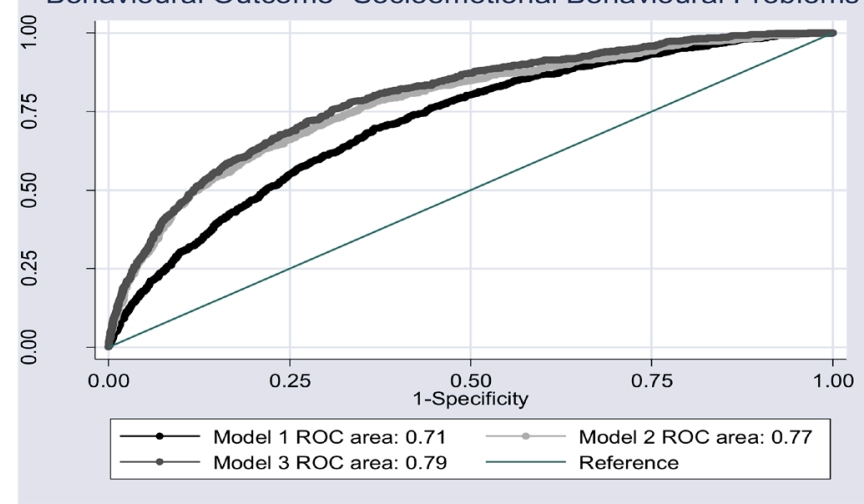

IDI (\%/p value): model 2 over model $16.26 /<0.001$, model 3 over model $22.01 / /<0.001$

Figure 2 AUC and IDI of language disability, overweight/obesity and socioemotional behaviour problems at age 11 for UK children. AUC, area under the curve; IDI, integrated discrimination improvement; ROC, receiver-operator characteristic.

With regard to the statistical selection method applied to develop model 3, the language disability and overweight/obesity models included 14 variables, and 22 variables were selected for the socioemotional behavioural problems model (figure 1). Figure 2 shows the ROC curve for each outcome in separate panels, with model 1 in black, model 2 in light grey and model 3 in dark grey: Language disability was identified with moderate discrimination ability for model 1 (AUROC: $0.7095 \%$ CI 0.68 to 0.72 ), model 2 (AUROC: 0.73 , 95\% CI 0.71 to 0.75 ) and model 3 (AUROC: $0.76,95 \%$ CI 0.74 to 0.78 ). Overweight/obesity was identified with poor discrimination in model 1 (AUROC: 0.66, 95\% CI 0.65 to 0.67 ) and moderate discrimination for models 2 (AUROC: $0.73,95 \%$ CI 0.72 to 0.74 ) and model 3 (AUROC: 
Table 1 Test properties of maximised cut-off probability for language disability, overweight/obese and socioemotional behavioural problems at age 11

\begin{tabular}{|c|c|c|c|c|c|c|c|c|c|}
\hline \multirow[b]{3}{*}{ Test properties } & \multicolumn{9}{|c|}{ Maximised cut-offs } \\
\hline & \multicolumn{3}{|c|}{ Cognitive language disability $(\%)^{*}$} & \multicolumn{3}{|c|}{ Physical overweight/obese (\%)† } & \multicolumn{3}{|c|}{ Behavioural socioemotional problems $(\%) \neq$} \\
\hline & Model 1 & Model 2 & Model 3 & Model 1 & Model 2 & Model 3 & Model 1 & Model 2 & Model 3 \\
\hline Sensitivity & 57.0 & 55.8 & 68.5 & 64.2 & 66.7 & 68.2 & 60.7 & 62.2 & 69.6 \\
\hline Specificity & 71.6 & 77.4 & 71.62 & 60.7 & 69.1 & 67.6 & 70.3 & 79.3 & 74.0 \\
\hline PPV & 12.7 & 15.2 & 14.89 & 37.6 & 44.3 & 43.7 & 15.4 & 21.0 & 19.2 \\
\hline NPV & 95.8 & 96.0 & 96.9 & 82.2 & 84.9 & 85.2 & 95.3 & 95.9 & 96.5 \\
\hline$\%$ of positives & 30.3 & 24.8 & 31.1 & 46.0 & 40.5 & 42.0 & 32.2 & 24.1 & 29.5 \\
\hline Correctly classified & 70.6 & 75.9 & 71.4 & 61.7 & 68.5 & 67.8 & 69.5 & 77.9 & 73.7 \\
\hline
\end{tabular}

Millennium Cohort Study, 2001-2012, UK (imputed data, $n=10262$ ).

${ }^{*}$ Maximised cut-offs used for language disability (model 1: 0.08 , model 2: 0.08, model 3: 0.07 ).

†Maximised cut-offs used for overweight/obese (model 1: 0.24 , model 2: 0.26 , model 3: 0.25 ).

‡Maximised cut-offs used for socioemotional behavioural problems (model 1: 0.09, model 2: 0.09, model 3: 0.08).

$\%$ of positives: total of children classified as positive, even if it is true or not; correctly classified: true positives plus true negatives; NPV, negative predictive value; PPV, positive predictive value.

$0.73,95 \%$ CI 0.72 to 0.74$)$. Socioemotional behavioural problems were also identified with moderate discrimination in all models (model 1: AUROC: $0.71,95 \%$ CI 0.69 to 0.73 ; model 2: AUROC: $0.77,95 \%$ CI 0.75 to 0.79 ; model 3: AUROC: 0.79 , $95 \%$ CI 0.77 to 0.80 , respectively). IDI indicated that model 2 resulted in a significant improvement in discrimination over model 1 , particularly for overweight/obesity and socioemotional behavioural problems with $8.14 \%$ and $6.26 \%$ more children being correctly reclassified by model 2 compared with model 1 , respectively. The IDI improvement was smaller for model 3 compared with model 2 for all outcomes, but remained significant (figure 2).

Sensitivity, specificity, positive predictive value, negative predictive value, percentage of positives and correctly classified for all models are shown in table 1 . Model 2 was the most accurate model for all outcomes, which means that this model had the best correct classification of children with health and development problems. Table 2 presents the multivariable associations between risk factors and language disability, overweight/ obesity and socioemotional behavioural problems at age 11, calibration and dominance analyses for model 2 (model with the best correct classification of children for all outcomes). Those results for models 1 and 3 can be found in the online supplementary material.

The Hosmer-Lemeshow goodness-of-fit tests indicate adequate calibration in model 2 for all outcomes (Hosmer-Lemeshow/p value: language disability 5.19/0.737; overweight/obesity 4.65/0.794; socioemotional behavioural problems, model 2 $14.42 / 0.071)$. Dominance analyses for model 2 showed that the top four most relevant factors for socioemotional behaviours at age 11 years were socioemotional behavioural problems at age 3 (0.502), smoking in pregnancy $(0.067)$, parental concerns about child speech at age $3(0.066)$ and neither parent in work (0.064). The most dominant factors for language disability at age 11 were naming and vocabulary disabilities at age $3(0.362)$, neither parent in work $(0.142)$, parental concerns about understanding of child speech at age $3(0.108)$ and socioemotional behavioural problems at age $3(0.094)$. For overweight/obesity at age 11 , overweight/obesity at age $3(0.589)$, maternal pre-pregnancy BMI indicating overweight/obese (0.291), smoking in pregnancy $(0.026)$ and greater deprivation of area of residence $(0.020)$ were the most important items.

Sensitivity analyses of AUROC not including prior measures of the outcome show similar findings to our main results, with moderate discrimination in models $2-3$ for socioemotional behavioural and language problems, but lower discrimination for obesity/overweight (about 68\% for models 2 and 3-see online supplementary material). In dominance analyses, when we remove prior measures of the relevant outcome, the second, third most influential variables and so on rise in the rank of importance (online supplementary material). Repeating the analysis including all of the variables from the ASQ in model 3 did not alter the model selection or change the results.

\section{DISCUSSION}

Using UK data from the MCS, we show that information collected in the first 3 years of life can be a potential tool to predict adverse health and developmental outcomes at age 11 with moderate accuracy. The discriminatory capacity of a model using data collected in maternity services in England is improved when updated with data routinely collected at $2-3$ years (particularly earlier measures of the relevant outcomes), but addition of wider set of perinatal, family/environmental and early childhood factors up to age 3 years did not alter risk prediction.

The first 3 years of life provide a unique opportunity to intervene and improve child development and subsequent adult outcomes. ${ }^{7}$ There has been a raft of policies promoting the benefits of early intervention, but the research base to support effective targeting of these initiatives is still emerging. Child health policy recommendations in the UK apply the principles of proportionate universalism, with universal services provided for all families and, in addition, progressively more intensive support targeted at those with greater need. ${ }^{67}$ In a technical sense, we would like to be able to find a set of characteristics (eg, maternal, partner, child and community) that accurately identify those children most at risk for poorer developmental outcomes, to help plan improved services for their future development.

While it is true that relative concentrations of poorer outcomes are higher in disadvantaged populations, to our knowledge, there has been little systematic work examining the extent to which these outcomes are predicted by risk factors earlier in the life course. The existing studies that have investigated this and have similarly demonstrated the utility of using data collected at birth to predict poor child health outcomes. Chittleborough and colleagues ${ }^{11}$ used a prospective, regional birth cohort in England to explore the predictive value of maternal age, compared with a model using six predictors (mother $<20$ 
Table 2 Multivariable associations between factors included in model 2 and language disability, overweight/obese and socioemotional behavioural problems at age 11

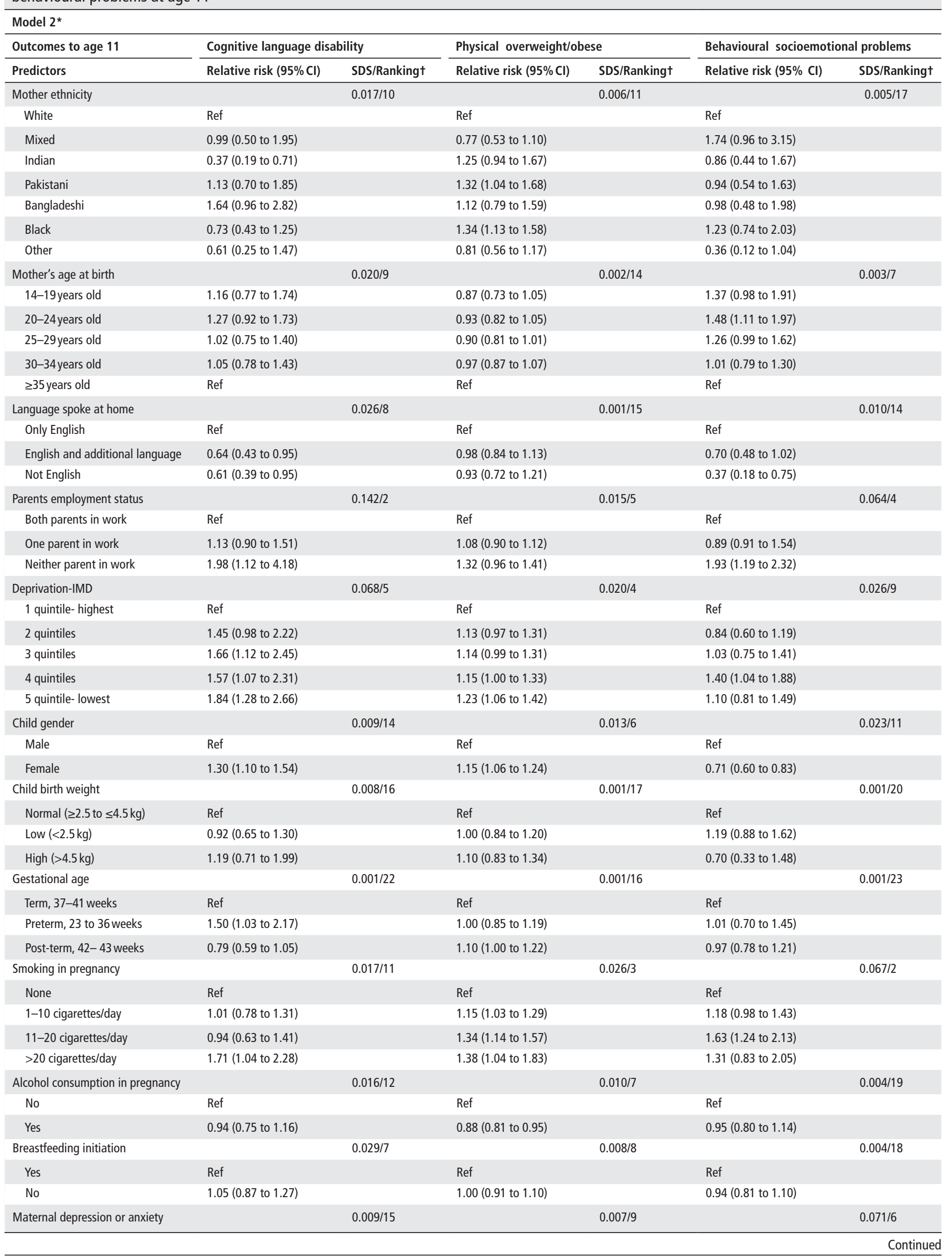


Table 2 Continued

\begin{tabular}{|c|c|c|c|c|c|c|}
\hline \multicolumn{7}{|l|}{ Model 2* } \\
\hline \multirow{2}{*}{$\begin{array}{l}\text { Outcomes to age } 11 \\
\text { Predictors }\end{array}$} & \multicolumn{2}{|c|}{ Cognitive language disability } & \multicolumn{2}{|c|}{ Physical overweight/obese } & \multicolumn{2}{|c|}{ Behavioural socioemotional problems } \\
\hline & Relative risk $(95 \% \mathrm{Cl})$ & SDS/Ranking $†$ & Relative risk $(95 \% \mathrm{Cl})$ & SDS/Ranking $†$ & Relative risk $(95 \% \mathrm{CI})$ & SDS/Ranking $\dagger$ \\
\hline $\begin{array}{l}\text { No } \\
\text { Yes }\end{array}$ & $\begin{array}{l}\text { Ref } \\
1.08(0.90 \text { to } 1.30)\end{array}$ & & $\begin{array}{l}\text { Ref } \\
1.06(0.98 \text { to } 1.16)\end{array}$ & & $\begin{array}{l}\text { Ref } \\
1.33(1.13 \text { to } 1.57)\end{array}$ & \\
\hline Type of delivery & & $0.001 / 19$ & & $0.002 / 12$ & & $0.001 / 22$ \\
\hline $\begin{array}{l}\text { Normal } \\
\text { Assisted (forceps, vacuum, breach) }\end{array}$ & $\begin{array}{l}\text { Ref } \\
1.17(0.80 \text { to } 1.70)\end{array}$ & & $\begin{array}{l}\text { Ref } \\
0.90(0.79 \text { to } 1.01)\end{array}$ & & $\begin{array}{l}\text { Ref } \\
1.00(0.71 \text { to } 1.40)\end{array}$ & \\
\hline Planned caesarean & 1.18 (0.88 to 1.58$)$ & & $1.06(0.93$ to 1.20$)$ & & 0.81 (0.63 to 1.04$)$ & \\
\hline Emergency caesarean & 1.03 (0.80 to 1.34$)$ & & 0.99 (0.89 to 1.11$)$ & & 1.07 (0.86 to 1.32$)$ & \\
\hline Other & $1.10(0.26$ to 4.61$)$ & & $0.82(0.47$ to 1.43$)$ & & $1.17(0.57$ to 2.37$)$ & \\
\hline Mother BMI before born & & $0.007 / 17$ & & $0.291 / 2$ & & $0.017 / 12$ \\
\hline Normal & Ref & & Ref & & Ref & \\
\hline Overweight/obese & 1.26 (1.05 to 1.52$)$ & & $1.85(1.70$ to 2.00$)$ & & 1.32 (1.13 to 1.53$)$ & \\
\hline Mother disability or illness & & $0.004 / 21$ & & $0.006 / 10$ & & $0.025 / 10$ \\
\hline No & Ref & & Ref & & Ref & \\
\hline Yes & $0.88(0.72$ to 1.07$)$ & & 1.07 (0.98 to 1.17$)$ & & $1.26(1.08$ to 1.46$)$ & \\
\hline Hearing problems age 3 & & $0.008 / 20$ & & $0.001 / 23$ & & $0.007 / 16$ \\
\hline No & Ref & & Ref & & Ref & \\
\hline Yes & 0.93 (0.64 to 1.36$)$ & & 1.12 (0.97 to 1.29$)$ & & 1.10 (0.83 to 1.46$)$ & \\
\hline Concern about child's speech age 3 & & $0.043 / 6$ & & $0.001 / 21$ & & $0.066 / 3$ \\
\hline No & Ref & & Ref & & Ref & \\
\hline Yes & 1.60 (1.23 to 2.08$)$ & & 1.01 (0.90 to 1.15$)$ & & 1.59 (1.30 to 1.95$)$ & \\
\hline Understands child's speech age 3 & & $0.108 / 3$ & & $0.001 / 20$ & & $0.046 / 5$ \\
\hline Always & Ref & & Ref & & Ref & \\
\hline Sometimes & 0.97 (0.64 to 1.47$)$ & & 0.75 (0.57 to 0.98$)$ & & $0.88(0.60$ to 1.30$)$ & \\
\hline Rarely & $2.16(1.35$ to 3.46$)$ & & $1.10(0.68$ to 1.76$)$ & & $1.08(0.63$ to 1.85$)$ & \\
\hline Walkup steps age 3 & & $0.011 / 13$ & & $0.002 / 13$ & & $0.010 / 15$ \\
\hline Yes & Ref & & Ref & & Ref & \\
\hline With help & 1.00 (0.57 to 1.76$)$ & & $0.79(0.59$ to 1.07$)$ & & $1.07(0.73$ to 1.57$)$ & \\
\hline No & 1.67 (1.08 to 2.58$)$ & & $1.28(1.00$ to 1.63$)$ & & 1.27 (0.86 to 1.88$)$ & \\
\hline Child disability or illness age 3 & & $0.004 / 18$ & & $0.001 / 22$ & & $0.031 / 8$ \\
\hline No & Ref & & Ref & & Ref & \\
\hline Yes & 1.09 (0.87 to 1.35$)$ & & $0.96(0.88$ to 1.05$)$ & & $1.36(1.16$ to 1.60$)$ & \\
\hline Naming vocabulary disability age 3 & & $0.362 / 1$ & & $0.001 / 19$ & & $0.015 / 13$ \\
\hline No language disability & Ref & & Ref & & Ref & \\
\hline Language disability & 2.51 (1.95 to 3.23$)$ & & $0.98(0.86$ to 1.13$)$ & & $1.10(0.83$ to 1.45$)$ & \\
\hline SDQ age 3 & & $0.094 / 4$ & & $0.001 / 18$ & & $0.502 / 1$ \\
\hline No related problems & Ref & & Ref & & Ref & \\
\hline Behavioural problems & 1.39 (1.12 to 1.72$)$ & & 0.98 (0.87 to 1.10$)$ & & 2.68 (2.22 to 3.23$)$ & \\
\hline BMI age 3 & & $0.001 / 23$ & & $0.589 / 1$ & & $0.001 / 21$ \\
\hline Normal weight & Ref & & Ref & & Ref & \\
\hline Overweight/obese & $0.92(0.73$ to 1.17$)$ & & $2.47(2.28$ to 2.67$)$ & & $1.05(0.88$ to 1.27$)$ & \\
\hline Hosmer-Lemeshow/p values $\ddagger$ & $5.19 / 0.737$ & & $4.65 / 0.794$ & & $14.42 / 0.071$ & \\
\hline
\end{tabular}

MCS, 2001-2012, UK (imputed data, $\mathrm{n}=10262$ ).

*Model 2 includes information collected in maternity services in England plus correspondent factors assessed in MCS at age 3 that are collected on 2.5 -year-old health check in England. tSDS and weighted ranking of predictive risk variables.

¥Calibration analyses.

BMI, body mass index; IMD, Index of Multiple Deprivation; MCS, Millennium Cohort Study; SDQ, Strengths and Difficulties Questionnaire; SDS, Standardised Dominance Statistic.

years, low maternal education, single parent, financial difficulties, depression, smoking in pregnancy) for child development outcomes up to age 5 years. Predictive capacity was improved in this study by including other data, but was still classified as poor $(A U R O C=0.67)$. The authors concluded that, even though maternal age is used to target early years child health programmes in many countries, these interventions will have little impact at a population level, since the majority of at risk children will miss out on intervention if young maternal age is the sole or main means of identifying eligibility for the programmes. ${ }^{11}$

A recent study from Australia used linked administrative perinatal datasets linked to data from the Australian Early Development Census to assess whether poor child development at age 5 could be predicted at a population level. ${ }^{12} \mathrm{~A}$ model with six perinatal characteristics (low maternal age, mother's marital status (never married, widowed, divorced or separated), mother 
and father's occupation (home duties, students, pensioners, unemployed), high number of previous pregnancies resulting in births $\geq 20$ weeks and smoking in second half of pregnancy) had poor discrimination for boys (AUROC $=0.68$ ) and moderate discrimination for girls (AUROC $=0.72$ ). The authors suggest that even with poor-moderate capacity of the models, if these six characteristics were used for targeting intensive support services and the programme targeted families with at least three of the six perinatal risk factors, approximately $10 \%$ of families in the population would be identified as needing an intensive intervention soon after birth. ${ }^{12}$ Building on these findings, our study shows that risk predictions were not substantially improved using a wider range of variables in the first 3 years of life and that these data also have moderate predictive value for outcomes at 11 years.

Socioeconomic factors and early measures of the relevant outcomes were the most important predictive indicators for child health and development at age 11 years. However, removing the early measure of the outcome from the analysis did not impact greatly on prediction, especially for language disabilities and socioemotional behavioural problems (as tested in our sensitivity analyses). Despite the high prevalence of overweight/obesity, it is to have been expected that predictive power for this outcome would be lower without age 3 years measurement, due to biological influences. ${ }^{25}$ Recent findings from predictive modelling studies in high-income countries, in the UK and Australia ${ }^{37}$ and in the USA ${ }^{38}$ corroborate the importance of social factors for later child health and development outcomes, even in high-income countries. Another study from Brazil (a middle-income country), using the 2004 Pelotas Birth Cohort, assessed a predictive model of early life factors for a cognitive outcome (low IQ) at age 6 years. Twelve risk factors were included in the final model and dominance analyses showed that social factors were the most important predictors. ${ }^{39}$

A strength of our study is the use of a large, contemporary UK cohort. A wide range of information is collected in the MCS, which allowed us to explore a large set of demographic, perinatal and early childhood risk factors. Measured BMI, validated assessments of language disability and socioemotional behavioural problems in children were also advantages. The MCS thus allowed us to consider what might be achieved through linkage of administrative datasets in the UK and to assess what added predictive value extra data collection might provide.

A limitation of our study is the lack of an external validation sample. In addition, missing data and attrition are common to all cohort studies, but the similar results in complete case and imputed datasets in our study offer reassurance that the risk of bias is minimised. We note that model 2 in our analysis included early measures of the prior problem, and it could be the case that much of the predictive value in the model could be explained by these early measures. However, repeating the analysis without these measures suggests that this is not the case (online supplementary material). We based most of our results on maternal self-reported data and decisions were made around categorising prediction variables. We have used cohort data from the nationally representative MCS and we expect that the predictors identified in the MCS would predict outcomes similarly in the general population. However, it is unclear the extent to which these models can be reproduced in routinely collected data. Further limitations include concerns about how similar the measures in the MCS are to those used in health services, since MCS data variables are aimed at research and to capture a picture of a representative sample of all UK children. Furthermore, we do not have detailed data on any intervention or specialised services that children may have accessed, that may have attenuated the associations in our study.

Further research is needed to assess the utility and impact of predictive risk models for child health and development outcomes in routine practice. We have used cohort data from the nationally representative MCS, but it is unclear the extent to which these models can be reproduced in routinely collected data. While many of the variables used in model 1 in our analysis should be available in routine data, other variables such as breastfeeding status and early measures of maternal mental health are more difficult to capture and may be of poor quality in routine data collection systems. Furthermore, we require a better understanding of how predictive risk modelling tools could be used in the context of specific child health systems, for instance, in the UK, what proportion of children would go on to receive specialist intervention; what proportion of those would benefit from this and what would be the magnitude of any benefits.

In the UK and the USA, there have been some attempts to target services on the basis of child and family characteristics, and our study provides evidence as to which variables are likely to be useful for this purpose in clinical and public health practice. ${ }^{40}{ }^{41}$ As many high-income countries collect these sort of data, it would be instructive to test how well they predict the same outcomes. The use of such tools raises ethical issues, for instance being labelled high risk could be stigmatising and any population level targeting approach would generate false positives (and false negatives), that would have opportunity costs for services locally. The implementation of risk prediction tools to guide policies would have to be carefully considered to ensure families were appropriately counselled and supported.

\section{CONCLUSION}

New child health datasets have been developed in England, but it remains a challenge to harness these population-level administrative datasets to improve outcomes for children. Our analysis shows that language disability, socioemotional behavioural problems and overweight/obesity in UK children aged 11 years can be predicted with moderate discrimination using data routinely collected in England. Addition of further variables identified in the literature that mostly are not routinely collect in health services does not add considerable improvement on discriminatory capacity of health and development problems in later

\section{What is already known on this subject}

- Early identification of children at risk of poor health and developmental outcomes is challenging, and some existing studies suggest that data routinely collected in health services could be better used for this purpose.

What this study adds

- We show that language disability, socioemotional behavioural problems and overweight/obesity are common in UK children aged 11 years and can be predicted with moderate discrimination using data routinely collected in the first 3 years of life. 
childhood. Further research is needed to identify what could increase the predictive power of these models at these and other ages in population-based databases such as MCS as well as assess how the dynamics of predictive algorithm models can be used in health services to identify children more likely to benefit from additional early years support.

Acknowledgements We would like to thank all the Millennium Cohort families for their participation, and the director of the Millennium Cohort Study and colleagues in the management team at the Centre for Longitudinal Studies, UCL Institute of Education.

Contributors VSS carried out the statistical analyses, drafted the initial manuscript, reviewed and revised the manuscript. AP and SH participated in the drafting of the initial manuscript, reviewed and revised the manuscript. BB participated in the drafting of the initial manuscript, reviewed and revised the manuscript. MW contributed to the conceptualisation of the study, participated in the drafting of the initial manuscript, reviewed and revised the manuscript. CL conceptualised and designed the study, participated in the drafting of the initial manuscript, reviewed and revised the manuscript. DT-R conceptualised and designed the study, coordinated, drafted the initial manuscript, reviewed and revised the manuscript. All authors approved the final manuscript as submitted and agree to be accountable for all aspects of the work.

Funding This work was supported by the UK Public Health Research Consortium (PHRC). The PHRC is funded by the Department of Health and Social Care Policy Research Programme. The views expressed in this paper are those of the authors and do not necessarily reflect those of the Department of Health and Social Care. Information about the wider programme of the PHRC is available from http://phrc Ishtm.ac.uk/. AP is funded by The Wellcome Trust (205412/Z/16/Z), the Medical Research Council (MC_UU_12017/13) and the Scottish Government Chief Scientist Office (SPHSU13). BB is supported by the National Institute for Health Research (NIHR) Collaboration for Leadership in Health Research and Care (CLAHRC NWC). The NIHR had no role in the study design, data collection and analysis, decision to publish or preparation of the article. This report is independent research arising from research supported by the NIHR. DTR is funded by the MRC on a Clinician Scientist Fellowship (MR/P008577/1)

\section{Competing interests None declared.}

Patient consent Not required.

Ethics approval Ethical approval for each wave of the MCS was granted by NHS Multicentre Research Ethics Committees. No further ethical approval was required for this secondary analysis of MCS data.

Provenance and peer review Not commissioned; externally peer reviewed.

Data sharing statement All MCS data used inthis analysis are available from UK Data Service, University of Essexand University of Manchester: http://doi.org/10. 5255/UKDA-SN-4683-4; http://doi.org/10.5255/UKDA-SN-5350-4; http://doi.org/10. 5255/UKDA-SN-7464-3.

Open access This is an open access article distributed in accordance with the Creative Commons Attribution 4.0 Unported (CC BY 4.0) license, which permits others to copy, redistribute, remix, transform and build upon this work for any purpose, provided the original work is properly cited, a link to the licence is given, and indication of whether changes were made. See: https://creativecommons.org/ licenses/by/4.0/.

\section{REFERENCES}

1 Department for Education. Statutory framework for the Early Years Foundation Stage: Setting the standards for learning, development and care for children from birth to five, 2014:1-33.

2 National Children's Bureau. The integrated review: Bringing together health and early education reviews at age two to two-and-a-half. London, 2015. http://www.ncb.org. uk/media/1201160/ncb_integrated_review_supporting_materials_for_practitioners_ march_2015.pdf. (accessed 21 Nov 2015).

3 Blades RGV, Wallace E, Loveless L, et al, 2014. Implementation study: Integrated review at 2-21/2 years - Integrating the early years foundation stage progress check and the healthy child programme health and development review. Department for Education https://http://www.gov.uk/government/publications/integrated-review-atage-2-implementation-study (accessed 21 Nov 2015).

4 Graham AMP. Early intervention: The next steps an independent report to her majesty's government. 2011 https://www.gov.uk/government/uploads/system/ uploads/attachment_data/file/284086/early-intervention-next-steps2.pdf.

5 Bricker D, Squires J, Mounts L. Ages and Stages Questionnaire: A parent-completed, child-monitoring system. Baltimore, MD: Paul H. Brookes, 1995.
6 Lynch JW, Law C, Brinkman S, et al. Inequalities in child healthy development: some challenges for effective implementation. Soc Sci Med 2010;71:1244-8.

7 Marmot M, Allen J, Goldblatt P, et al. Fair society, healthy lives: strategic review of health inequalities in England post. London, UK: UK Department of Health, 2010.

8 D'Agostino RB, Vasan RS, Pencina MJ, et al. General cardiovascular risk profile for use in primary care: the Framingham Heart Study. Circulation 2008;117:743-53.

9 Lloyd-Jones DM. Cardiovascular risk prediction: basic concepts, current status, and future directions. Circulation 2010;121:1768-77.

10 Nelson SM, Lawlor DA. Predicting live birth, preterm delivery, and low birth weight in infants born from in vitro fertilisation: a prospective study of 144,018 treatment cycles. PLoS Med 2011;8:e1000386.

11 Chittleborough CR, Lawlor DA, Lynch JW. Young maternal age and poor child development: predictive validity from a birth cohort. Pediatrics 2011;127:e143 6-e1444.

12 Chittleborough CR, Searle AK, Smithers LG, et al. How well can poor child development be predicted from early life characteristics? A whole-of-population data linkage study. Early Child Res Q 2016;35:19-30.

13 Connelly R, Platt L. Cohort profile: UK Millennium Cohort Study (MCS). Int J Epidemiol 2014:43:1719-25.

14 Elliott C. The British Ability Scales II. Windsor, Berkshire: UK: NFER-NELSON Publishing Company, 1996

15 Elliott CD, Smith P, McCulloch K. Technical manual British Ability Scales II. Windsor. Berkshire: NFER-NELSON Publishing Company, 1997.

16 Reilly S, Wake M, Ukoumunne OC, et al. Predicting language outcomes at 4 years of age: findings from Early Language in Victoria Study. Pediatrics 2010;126:e153 0-e1537.

17 Beitchman JH, Wilson B, Johnson CJ, et al. Fourteen-year follow-up of speech/ language-impaired and control children: psychiatric outcome. J Am Acad Child Adolesc Psychiatry 2001;40:75-82.

18 Justice LM, Bowles RP, Pence Tumbell KL, et al. School readiness among children with varying histories of language difficulties dren with varying histories of language difficulties. Dev Psychol 2009;45:460-76.

19 Cole TJ, Lobstein T. Extended international (IOTF) body mass index cut-offs for thinness, overweight and obesity. Pediatr Obes 2012;7:284-94.

20 Goodman R. The strengths and difficulties questionnaire: A research note. J Child Psychol Psychiatry 1997;38:581-6.

21 Hope S, Pearce A, Whitehead M, et al. Family employment and child socioemotional behaviour: longitudinal findings from the UK Millennium Cohort Study. J Epidemiol Community Health 2014;68:950-7.

22 Wickham S, Whitehead M, Taylor-Robinson D, et al. The effect of a transition into poverty on child and maternal mental health: a longitudinal analysis of the UK Millennium Cohort Study. Lancet Public Health 2017;2:e141-e148.

23 Maternity Services Monthly Statistics, England - February 2017, Experimental statistics. National Health Service NHS Digital 2017 http://www.digital.nhs.uk/ catalogue/PUB30017 (accessed on 14 Aug 2017).

24 Weng SF, Redsell SA, Swift JA, et al. Systematic review and meta-analyses of risk factors for childhood overweight identifiable during infancy. Arch Dis Child 2012.

25 Hobcraft JN, Kiernan KE. Predictive factors from age 3 and infancy for poor child outcomes at age 5 relating to children's development, behaviour and health: evidence from the Millennium Cohort Study [Internet]. University of York: York, 2010. http:// www.york.ac.uk/media/spsw/documents/research-and-publications/HobcraftKier nan2010PredictiveFactorsChildrensDevelopmentMillenniumCohort.pdf. (cited 28 May 2017).

26 Kiernan KE, Mensah FK. Maternal indicators in pregnancy and children's infancy that signal future outcomes for children's development, behaviour and health: evidence from the Millennium Cohort Study. University of York: York, 2010.

27 Borrell-Porta M, Cooper K, Font JC, et al. Children's wellbeing and development outcomes for ages 5, 7 and 11, and their predictors -Final report: London School of Economics (Department of Social Policy and LSE Enterprise), 2017.

28 Walker SP, Wachs TD, Grantham-McGregor S, et al. Inequality in early childhood: risk and protective factors for early child development. The Lancet 2011;378:1325-38.

29 Spencer N, Thanh TM, Louise S. Low income/socio-economic status in early childhood and physical health in later childhood/adolescence: a systematic review. Matern Child Health J 2013:17:424-31.

30 Hanley JA, McNeil BJ. The meaning and use of the area under a receiver operating characteristic (ROC) curve. Radiology 1982;143:29-36.

31 Hajian-Tilaki K. Receiver Operating Characteristic (ROC) Curve Analysis for Medical Diagnostic Test Evaluation. Caspian J Intern Med 2013;4:627-35.

32 Pepe MS, Janes $\mathrm{H}$, Longton $\mathrm{G}$, et al. Limitations of the odds ratio in gauging the performance of a diagnostic, prognostic, or screening marker. Am J Epidemiol 2004:159:882-90.

33 Pencina MJ, D'Agostino RB, D'Agostino RB, et al. Evaluating the added predictive ability of a new marker: from area under the ROC curve to reclassification and beyond. Stat Med 2008;27:157-72.

34 Hosmer DW, Lemesbow S. Goodness of fit tests for the multiple logistic regression model. Commun Stat Theory Methods 1980;9:1043-69. 
35 Johnson JW. A heuristic method for estimating the relative weight of predictor variables in multiple regression. Multivariate Behav Res 2000;35:1-19.

36 Royston P. Multiple imputation of missing values. The Stata Journal 2004;4:227-41.

37 Nelson BB, Dudovitz RN, Coker TR, et al. Predictors of poor school readiness in children without developmental delay at age 2. Pediatrics 2016:138:e20154477.

38 Smithers LG, Chittleborough CR, Stocks N, et al. Can items used in 4-year-old wellchild visits predict children's health and school outcomes? Matern Child Health J 2014; 18:1345-53.
39 Camargo-Figuera FA, Barros AJD, Santos IS, et al. Early life determinants of low IQ at age 6 in children from the 2004 Pelotas Birth Cohort: a predictive approach. BMC Pediatr 2014;14:308.

40 Chung EK, Siegel BS, Garg A, et al. Screening for social determinants of health among children and families living in poverty: A guide for clinicians. Curr Prob/ Pediatr Adolesc Health Care 2016;46:135-53.

41 Garg A, Toy S, Tripodis Y, et al. Addressing social determinants of health at well child care visits: a cluster RCT. Pediatrics 2015;135:e296-e304. 